\title{
Histopathological alterations in irritable bowel syndrome
}

\author{
Richard H Kirsch and Robert Riddell \\ Department of Pathology and Laboratory Medicine, Mount Sinai Hospital and University of Toronto, Toronto, \\ Ontario, Canada
}

\begin{abstract}
Irritable bowel syndrome is a common disorder defined by a symptom complex including abdominal pain and altered bowel habit. The etiopathogenesis appears to be multifactorial and to involve altered gastrointestinal motor function, enhanced perception of visceral stimuli and psychosocial factors. More recently a role for mucosal immune activation has been suggested. Routine histologic examination reveals no mucosal abnormality in the majority of cases but quantitative histological, immunohistochemical and ultrastructural analyses reveal subtle morphologic changes involving lymphocytes, mast cells, enterochromaffin cells and enteric nerves. The recent appreciation of these changes has led to new hypotheses linking central and enteric nervous systems to immune processes. This review highlights the spectrum of morphologic changes that occur in irritable bowel syndrome, examines their relationship to the pathophysiology of irritable bowel syndrome and considers their relevance to daily pathology practice.
\end{abstract}

Modern Pathology (2006) 19, 1638-1645. doi:10.1038/modpathol.3800704; published online 29 September 2006

Keywords: irritable bowel syndrome; pathology; chronic inflammation; mast cell; enterochromaffin cell; enteric nerve

Irritable bowel syndrome (IBS) is the most common gastrointestinal disorder encountered in general and gastroenterology practice. ${ }^{1,2}$ It affects $10-20 \%$ of adolescents and adults in western societies ${ }^{3}$ and is the commonest cause of recurrent abdominal pain in children. ${ }^{4}$ IBS is defined by a symptom complex which includes abdominal pain and altered bowel function in the form of diarrhea, constipation, a sensation of fullness following evacuation, bloating or the passage of mucus per rectum. There are no physical or laboratory findings which are specific for IBS and the diagnosis is therefore based on symptomatology. To standardize the diagnosis of IBS, several symptom-based diagnostic criteria have been established over the last two decades including the Manning, Rome I, Rome II and, most recently, Rome III criteria. ${ }^{5}$ The Rome criteria have shown a reasonable sensitivity and specificity for IBS. In patients without atypical symptoms (such as loss of weight, fever, rectal bleeding and nocturnal awakening due to pain) the Rome criteria have a positive predictive value of approximately $98 \%$ for IBS, that

Correspondence: $\mathrm{Dr}$ RH Kirsch, MD, PhD, Department of Pathology and Laboratory Medicine, Mount Sinai Hospital, 600 University Avenue, Toronto, Ontario, Canada M5G 1X5.

E-mail: rkirsch@mtsinai.on.ca

Received 10 July 2006; revised and accepted 23 August 2006; published online 29 September 2006 is additional investigations will yield an alternative diagnosis in only $2 \%$ of cases. ${ }^{1,6}$

IBS has been subclassified into diarrhea predominant, constipation predominant and mixed subtypes. In addition, a subset of patients have associated extraintestinal symptoms including fatigue, fibromyalgia, urinary frequency and headache. ${ }^{6}$ A small but significant proportion of IBS of patients report onset of IBS symptoms following an episode of acute gastroenteritis (post-infectious IBS, PI-IBS). ${ }^{7-9}$ PI-IBS has been reported following Shigella, ${ }^{8}$ Salmonella, ${ }^{9,10}$ and Campylobacter jejuni ${ }^{7,11}$ infection. A unifying hypothesis to explain the pathogenesis of IBS remains elusive. Alterations in gastrointestinal motor function, enhanced visceral perception of painful stimuli and psychosocial factors are considered key contributors to symptom generation in IBS. ${ }^{12,13}$ Alterations in gastrointestinal motor function, enhanced visceral perception of painful stimuli and psychosocial factors are considered key contributors to symptom generation in IBS. ${ }^{12,13}$ More recently recognized factors include reduced ability to expel intestinal gas, altered central processing of afferent signals and intestinal inflammation. ${ }^{13}$ While routine histologic examination reveals no significant colonic mucosal abnormality in the majority of these patients, recent quantitative histological, immunohistochemical and ultrastructural analyses provide evidence of 
subtle morphologic changes in these patients. The recognition of these changes has led to new hypotheses linking central and enteric nervous systems to immune processes. This review highlights the spectrum of histologic changes that occur in IBS, examines their relationship to the pathophysiology of IBS and considers their relevance, if any, to daily pathology practice.

\section{Colonoscopy in IBS}

The American Gastroenterological Association recommends colonoscopy only in those patients with presumed IBS who are over 50 years of age or who have symptoms raising the possibility of another disease, in particular diarrhea predominance and/or weight loss. ${ }^{14}$ Therefore, in practice, only a small minority of patients with IBS will undergo colonoscopy and biopsy. Owing of its high prevalence, however, IBS still accounts for the majority of colonoscopic biopsies seen by many gastrointestinal pathologists. ${ }^{15}$ Most of these biopsies will be either normal or near to normal on routine histological examination. From a clinical standpoint, a normal pathology result therefore provides valuable information to the physician who is suspecting a diagnosis of IBS. It is thus important for pathologists to be aware of variations of normal as well as of artifacts that may result from bowel preparations or the biopsy procedure, and not to report these as abnormal. These changes will therefore be highlighted prior to reviewing the subtle morphologic changes recently reported in IBS.

\section{The spectrum of normal in colonic biopsies}

Variations exist in the normal histology of the right and left colon and these reflect the different functions of absorption and lubrication respectively. ${ }^{16}$ The crypts of the cecum and right colon show a predominance of absorptive cells over goblet cells which should not be mistaken for mucin depletion. The lamina propria of this region is more cellular than the left colon and rectum and includes lymphocytes, plasma cells (which may be basal) and eosinophils. Lamina propria cellularity in the left colon is much lower with fewer eosinophils and only superficial plasma cells. An age related decline in lymphocytes and mast cells has recently been reported in normal large bowel mucosa. ${ }^{16}$ Paneth cells are normally present in the mucosa of the cecum and right colon, but when seen in the left colon represent a metaplasia, usually in response to chronic inflammation. ${ }^{15,17}$ Lymphoid aggregates are a normal component of colorectal mucosa and are frequently associated with branching of overlying crypts. ${ }^{17}$ Aggregates of muciphages are commonly observed in colorectal mucosa, being present in $40 \%$ of normal rectal biopsies. ${ }^{18}$ They probably are a result of clinically insignificant damage to the crypt epithelium which may occur during stool passage or subclinical infection. Bowel preparations may induce a number of histologic changes. Hypertonic enemas, frequently administered prior to sigmoidoscopy, can induce surface epithelial injury including mucin depletion, mild neutrophilic infiltration and loss of surface epithelium as well as edema and hemorrhage in the lamina propria. ${ }^{19}$ Sodium phosphate preparations, the preferred preparations for full colonoscopy in adults, can induce aphthouslike lesions which histologically are associated with large lymphoid aggregates or less commonly edema, hemorrhage or acute inflammation. ${ }^{20-22}$ These preparations may also induce foci of neutrophilic cryptitis (focal active colitis) which is thought to occur in approximately $3 \%$ of patients. ${ }^{22}$ Tissue trauma induced by biopsy forceps can result in mucosal hemorrhage or extraction of epithelium from crypts. If this involves a large area, it may be mistaken for ischemic injury. ${ }^{15}$

\section{Histological changes in IBS}

The subtle morphologic changes reported in the intestinal mucosa in IBS involve chronic inflammatory cells, mast cells, enteroendocrine cells and enteric nerves, each of which is discussed below and summarized in Table 1.

\section{Chronic Inflammatory Cells}

Several investigators have demonstrated increased numbers of chronic inflammatory cells in the colonic mucosa of patients with IBS. ${ }^{11,12,23-27}$ In most studies, quantitative immunohistochemical analyses have been required to unmask these increases ${ }^{11,23-27}$ which are not usually apparent on routine histological evaluation. Both the lamina propria $^{23-25}$ and surface and crypt epithelium ${ }^{11,23,27}$ have been shown to contain increased numbers of T-lymphocytes in IBS. Such increases have been reported to occur in both PI-IBS ${ }^{11,12,24,25}$ and non-PIIBS. ${ }^{23,25}$ The diarrhea predominant form IBS is reported to be associated with a greater increase in mucosal T-lymphocytes than the constipation predominant form. ${ }^{23}$ Reported increases in mucosal lymphocytes range from 20 to $100 \%$ and 80 to $250 \%$, for lamina propria and epithelial lymphocytes, respectively. Such variations are likely to reflect differences in subpopulations of IBS patients studied and differences in the sites of biopsy. A marked increase in lamina propria inflammatory cells expressing CD25, a component the interleukin 2 receptor and a marker of immune activation, has recently been reported in colonic biopsies from patients with IBS. ${ }^{23}$ This was present in almost $90 \%$ of patients and was not dependent on the dominant symptom profile of IBS patients or a preceding episode of gastroenteritis. 
The histologic changes of PI-IBS following $C$. jejuni gastroenteritis have been well described. ${ }^{11}$ At 2 weeks postinfection, the mucosa in most cases has returned to normal by both macroscopic and conventional histologic assessment. However, quantitative histology reveals evidence of ongoing inflammation, which gradually decreases over the following 3 months. Inflammatory changes persist at 1 year in a small subgroup of these patients who also have clinical features fulfilling the Rome II criteria for IBS. These patients exhibit greater IL-1 $\beta$ mRNA expression, both during and after the infection, compared with individuals who do not develop IBS after an episode of gastroenteritis. ${ }^{12}$ In addition, patients with PI-IBS have recently been shown to have greater IL- $1 \beta$ mRNA expression than patients with non-PI-IBS, ${ }^{8}$ consistent with immune activation in this subset of patients.

\section{Mast Cells}

In 1962, Hiatt and $\mathrm{Katz}^{28}$ reported increased numbers of mast cells in the muscularis propria of four patients with 'spastic colitis'. These findings were not pursued by other investigators, perhaps due to the unavailability of full thickness colonic specimens in IBS patients. It is only the last decade that has seen a renewed interest in the association of mast cells in IBS..$^{8,25,29-32}$ In 1993, Weston et al ${ }^{32}$ demonstrated a marked increase in mast cell density in terminal ileum biopsies from patients with IBS compared to controls, a finding later confirmed by others. ${ }^{8}$ This increase was most marked in the diarrhea predominant subgroup ${ }^{32}$ but was present both in PI- and non-PI-IBS. ${ }^{8}$ Subsequent studies have demonstrated increased mast cell density in the cecal mucosa of IBS patients. ${ }^{29,31}$ While several investigators have failed to demonstrate increased numbers of mast cells in colorectal biopsies from patients with IBS, ${ }^{8,24,31,33,34}$ recent studies using sensitive techniques, such as electron microscopy and/or immunohistochemistry combined with computer assisted morphometry, have demonstrated an increased mast cell density in the descending colon, ${ }^{30}$ caecum and rectum. ${ }^{29}$ Immunoenzymatic studies have demonstrated increased mucosal mast cell tryptase content as well as increased spontaneous release of tryptase and histamine in IBS patients compared to controls. ${ }^{30}$ Ultrastructural studies have shown increased numbers of degranulating mast cells in IBS patients compared to controls $^{29,30}$ as well as increased numbers of mast cells in proximity to enteric nerves in the rectum, ${ }^{29}$ descending colon, ${ }^{30}$ caecum $^{29}$ and terminal ileum. ${ }^{8}$

Mast cells may be central in the strong association between interstitial cystitis (IC) and IBS, both of which are exacerbated by stress. Bladder and colon biopsies from a patient with both IC and IBS were showed an increase in both bladder and colonic mast cells, the latter mostly close to substance P-positive nerves. ${ }^{35}$ 


\section{Enterochromaffin Cells}

Increased numbers of enterochromaffin cells (EC) have been reported in rectal biopsies from patients with PI-IBS ${ }^{11,24,25}$ but not in non-PI-IBS. ${ }^{25}$ A fivefold increase in synaptophysin positive EC cells has been reported in rectal biopsies 2 weeks following $C$. jejuni infection. ${ }^{11}$ In these patients, EC numbers decreased gradually in biopsies taken at 6 and 12 weeks. ${ }^{11}$ However, in a small subset of patients who remained symptomatic at 1 year postinfection, rectal biopsies showed persistently elevated EC levels in the range seen at 2 weeks postinfection. ${ }^{7,11}$ These levels were similar to those seen in a group of PI-IBS patients recruited from an outpatient clinic. ${ }^{11}$ The profile of secretory granules also changed significantly postinfection, being 5-hydroxytryptamine (5-HT, serotonin) predominant 3 months following Campylobacter infection compared to peptide YY (PYY) predominant in normal controls. ${ }^{7}$ However, EC cells from patients who developed PI-IBS showed similar 5-HT/PYY ratios to those of nonPI-IBS patients and normal controls. ${ }^{25}$

\section{Enteric Nerves}

Increased numbers of nerve fibres staining positively for neurone specific enolase, substance P and 5-HT (but not calcitonin gene-related peptide) have been demonstrated in biopsies from the terminal ileum and rectosigmoid in patients with both PIand non-PI-IBS. ${ }^{8}$ In addition, positively stained nerve fibres around mast cells are reported to be significantly increased in density in IBS patients compared to controls. ${ }^{8}$ The distance between axonal fibres of the enteric nervous system and inflammatory cells, including mast cells ${ }^{8,29,30}$ and lymphocytes, ${ }^{36,37}$ is reported to be decreased in patients with IBS compared to controls. A study in evaluating full thickness jejunal biopsy specimens in 10 patients with severe IBS, reported striking neuronal changes including lymphocytic infiltration of the myenteric plexus (9/10 patients) and neuronal degeneration (7/10 patients). ${ }^{27}$ Mast cells were not identified in the vicinity of the myenteric plexus. An editorial expressed caution in the interpretation of these findings due to the small patient numbers, inclusion of only severe cases of IBS, suboptimal controls and the use of a scoring system for myenteric plexus associated lymphocytes that might exaggerate any differences present. ${ }^{38}$

\section{Morphologic changes and pathophysiology of IBS}

The recently documented low-grade inflammatory changes outlined above suggest that immune activation may, at least in part, play a role in the pathogenesis of IBS. The reported increases in inflammatory cells, although modest, could poten- tially be of pathophysiological significance if accompanied by similar increases in cytokine production. The recent documentation of increased numbers of immune cells expressing CD25, a marker of immune cell activation, ${ }^{23}$ provides functional evidence of immune activation in IBS. CD25 + cells are considered important regulators of intestinal inflammation, and it has been speculated that they may play a role in downregulating the inflammatory process in IBS. ${ }^{39}$ Further evidence for immune cell activation in IBS includes upregulation of IL-1- $\beta$ mRNA which has been documented in patients with PI-IBS ${ }^{8,40}$ but not in non-PI-IBS. ${ }^{8}$ This may reflect different pathogenetic mechanisms in these two subsets of patients.

The role of psychological stress is well recognized in the etiopathogenesis of IBS and there is growing evidence of interplay between immune and central nervous systems. Animal studies suggest that stress may enhance responsiveness to inflammatory stimuli in the gut, while inflammatory processes in the gut may influence behavior and brain function. ${ }^{41-44}$ The close proximity of chronic inflammatory cells to enteric nerves in the mucosa ${ }^{36,37}$ and muscularis externa $^{27}$ of patients with IBS, provides an interface for direct interaction between cells and the enteric nervous system. Potential mechanisms of immune activation in IBS that have been suggested include a previous episode of gastroenteritis, alterations in intestinal microflora, undiagnosed food allergies and genetic factors. ${ }^{45}$

Reports of increased mast cells numbers, ${ }^{8,25,29-32}$ increased mast cell degranulation, ${ }^{29,30}$ increased spontaneous release of mast cell tryptase and histamine $^{30}$ and increased proximity of MC to enteric nerves ${ }^{8,29,30}$ in IBS suggest a role for mast cells in the disturbed sensorimotor function characteristic of this condition. The proximity of mast cells to enteric nerves suggests that mast cell mediators have increased potential to activate enteric neurons. Indeed, the latter has been reported to correlate with the severity or frequency of abdominal pain or discomfort in patients with IBS. ${ }^{30}$ Mast cell mediators such as tryptase and histamine are known to activate enteric neurons leading to abnormal secretomotor function and visceral hypersensitivity. ${ }^{46,47}$ Conversely, substance $\mathrm{P}$ in low concentrations has been shown to alter mast cell excitability and can directly modulate mast cell function following release from nerves. ${ }^{48}$ The cause of mast cell infiltration and degranulation in IBS remains uncertain, but past episodes of infectious enteritis, ${ }^{7,11}$ undiagnosed food allergies ${ }^{49}$ and stress ${ }^{50}$ may contribute.

The modest increase in EC cell numbers reported in PI-IBS ${ }^{7,11,24,25}$ may have pathophysiologic significance. EC cells constitute the bulk of the body's 5HT stores ${ }^{13}$ and there is evidence of increased 5-HT release in patients with IBS. ${ }^{51,52}$ Enteric nerves and sensory afferents contain a number of receptors for $5 \mathrm{HT}^{53,54}$ and the prokinetic and secretory effects of 
5HT may underlie the diarrhea and loose stools in IBS. $^{25,54}$ In addition to its prokinetic effects, 5-HT may exert pro-inflammatory effects via 5-HT(2) receptors on vascular endothelial cells facilitating recruitment of additional T-lymphocytes. ${ }^{25}$ The mechanism underlying the increase in EC cells in IBS has not been elucidated although lymphocytederived cytokines or prostaglandins have been shown to induce increased EC numbers in animals. ${ }^{54} \mathrm{~A}$ decrease in serotonin transporter has been reported in association with EC hyperplasia in a mouse model of PI-IBS and this may serve to further enhance mucosal 5-HT availability. ${ }^{55}$ Anti5-HT(3) receptor antagonists have proved useful in the treatment of diarrhea predominant IBS, while the constipation predominant form may respond to 5HT(4) receptor agonists. ${ }^{56,57}$ In rare cases 5 -HT(3) receptor antagonist has been associated with colon ischemia. ${ }^{58}$ Interestingly, the rate of colon ischemia among patients carrying a diagnosis of IBS is over three times higher than that of the general population. ${ }^{59}$ This raises the question of whether 5-HT(3) receptor antagonists may really potentiate pre-existing ischemia or cause it de novo.

The reported increase in mucosal nerve fibres, ${ }^{8}$ increased proximity between nerve fibres and mast cells $^{8,13,29}$ and lymphocytes ${ }^{36,37}$ and lymphocytic infiltration and neuronal degeneration of the myenteric plexus ${ }^{27}$ in IBS provide a morphologic basis for a neuro-immune interaction. This close association between inflammatory cells and nerves may, at least in part, reflect plasticity of intestinal mucosal nerves $^{60}$ as regenerating nerves are reported to contact mast cells more frequently. ${ }^{61}$ Such nerve plasticity is well documented in the setting of intestinal inflammation. ${ }^{60}$ The ability of mucosal inflammation to alter enteric nerve and smooth muscle function is well established. ${ }^{62}$ The potential mechanisms facilitating these neuro-immune interactions, including both inflammatory and neural mediators have been discussed.

Finally, it should be emphasized that the precise relationship between the reported histopathologic changes in IBS and its pathogenesis remains to be defined. While a number of hypotheses link reported histologic changes to the pathogenesis of IBS, it remains possible that many such changes are unrelated to the pathogenesis of IBS and could be due to other factors such as associated motility disturbances (which may be particularly relevant in the constipation predominant form of IBS).

\section{Normal and minimally inflamed mucosal biopsies: more than meets the eye?}

The recent advances in our understanding of the immunopathology of IBS highlight the role of quantitative analyses in unmasking subtle changes in apparently normal mucosa. The concept of pathology that is subtle or unapparent on routine assessment is not unique to IBS. Quantitative analyses have uncovered subtle mucosal abnormalities in other conditions including food allergy, ${ }^{63}$ regressive autism, ${ }^{64-67}$ developmental disorders ${ }^{64}$ and Brainerd diarrhea. ${ }^{68}$ For example, there is an emerging pattern of mucosal immunopathology in a subpopulation of children with regressive autism who also have gastrointestinal symptoms. ${ }^{64-67}$ Histologically, the changes are fairly subtle and, apart from ileal-lymphoid-nodular hyperplasia, these patients have variable, non-specific inflammatory mucosal changes which are easily overlooked on routine assessment. However, immunohistochemical studies have demonstrated significant increases in CD8 and $\gamma \delta$ T-cells in the colonic mucosa of these patients. ${ }^{65}$ Moreover, flow cytometry of duodenal, ileal and colonic mucosal biopsies have shown increases in $\mathrm{CD} 3+, \mathrm{CD} 4+, \mathrm{CD} 8+$ and $\mathrm{CD} 19+$ intraepithelial lymphocytes as well as lamina propria CD3 + cells in these patients compared to controls. ${ }^{66}$ A higher proportion of CD3 + cells are positive for $\mathrm{TNF} \alpha$, IL-2, IFN $\gamma$ and IL-4 in these patients than in controls while a smaller proportion are IL-10 positive. ${ }^{67}$ Interestingly, CD4 + intraepithelial lymphocytes and lamina propria CD19+ B-cells are increased compared to non-autistic controls with mucosal inflammation (including a group of inflammatory bowel disease patients), suggesting a novel pattern of mucosal immunopathology. ${ }^{66}$ Similarly, morphometry has proved useful in children with multiple food allergies and has revealed subtle villous abnormalities which are not obvious on routine assessment. ${ }^{63}$ Finally, subtle morphologic alterations have been described in colonic biopsies from patients with Brainerd diarrhea, a term used to describe outbreaks of chronic watery diarrhea of unknown etiology associated with an abrupt onset and a prolonged course. ${ }^{68}$ Colonic biopsies from such patients are reported to show an intraepithelial lymphocytosis which may be subtle or can approach levels seen in lymphocytic and collagenous colitis. In contrast to the latter conditions, the surface epithelium in patients with Brainerd diarrhea retains its tall columnar appearance and there is no thickening of the subepithelial collagen plate. ${ }^{68}$ There may be a degree of overlap between Brainerd diarrhea and PI-IBS as both conditions have a presumed infectious etiology, can persist for more than a year and are often associated with an intra-epithelial lymphocytosis. The precise relationship between these two conditions remains to be elucidated. In summary, it appears that routine histopathological assessment may provide a limited view of mucosal abnormalities and, in some instances, may underestimate the changes present. Although there is currently no rationale for the use of immune markers in the routine assessment of gastrointestinal mucosal biopsies, it is possible that this may change as new immunopathologic data emerges. 


\section{Conclusion}

IBS is a symptom complex including abdominal pain/discomfort and altered bowel function and is heterogeneous with respect to aetiopathogenesis, clinical presentation and histopathology. Alterations in gastrointestinal motor function, increased visceral perception, altered central processing of afferent signals and psychosocial factors are thought to underlie symptom generation in IBS. Recent morphologic studies have seen enteric immune activation and altered neuro-immune interactions receiving increasing attention. Quantitative analyses using immunohistochemistry, morphometry or electron microscopy have shown unequivocal increases in enteric mucosal chronic inflammatory cells, mast cells and EC cells in IBS, despite apparently normal morphology on routine examination. There is also evidence of immune cell activation, mast cell degranulation and neuro-immune interactions. Such morphologic changes, although modest, may have pathophysiological significance in IBS. However, a definitive cause-effect relationship between minimal inflammation and IBS remains to be demonstrated.

Do the reported morphologic changes in IBS have applicability to daily pathology practice? Current data would indicate not. The overlap between patient and control groups, the need for appropriately matched controls, and laborious quantitative methods required to demonstrate abnormalities and non-specificity of the inflammatory changes, all pose major limitations. However, these reported changes do alter the way we view IBS and highlight the role of quantitative analyses in unmasking subtle changes in apparently normal tissue.

Finally, the value of recognizing and reporting a 'normal' or 'near normal' colonoscopic biopsy must be emphasized since this remains important in the diagnosis of the IBS in patients undergoing colonoscopic biopsy.

\section{References}

1 Olden KW. Diagnosis of irritable bowel syndrome. Gastroenterology 2002;122:1701-1704.

2 Mitchell CM, Drossman DA. Survey of the AGA membership relating to patients with functional gastrointestinal disorders. Gastroenterology 1987;92:1282-1284.

3 Ringel Y, Drossman DA. Irritable bowel syndrome: classification and conceptualization. J Clin Gastroenterol 2002;35:S7-S10.

4 El-Matary W, Spray C, Sandhu B. Irritable bowel syndrome: the commonest cause of recurrent abdominal pain in children. Eur J Pediatr 2004;163:584-588.

5 Lembo TJ, Fink RN. Clinical assessment of irritable bowel syndrome. J Clin Gastroenterol 2002;35: S31-S36.

6 Drossman DA. The functional gastrointestinal disorders and the Rome III process. Gastroenterol 2006;130: 1377-1390.
7 Spiller RC. Postinfectious irritable bowel syndrome. Gastroenterol 2003;124:1662-1671.

8 Wang LH, Fang XC, Pan GZ. Bacillary dysentery as a causative factor of irritable bowel syndrome and its pathogenesis. Gut 2004;53:1096-1101.

9 Spiller R, Campbell E. Post-infectious irritable bowel syndrome. Curr Opin Gastroenterol 2006;22:13-17.

10 McKendrick MW. Post Salmonella irritable bowel syndrome-5 year review. J Infect 1996;32:170-171.

11 Spiller RC, Jenkins D, Thornley JP, et al. Increased rectal mucosal enteroendocrine cells, T lymphocytes, and increased gut permeability following acute Campylobacter enteritis and in post-dysenteric irritable bowel syndrome. Gut 2000;47:804-811.

12 Gwee KA, Leong YL, Graham C, et al. The role of psychological and biological factors in postinfective gut dysfunction. Gut 1999;44:400-406.

13 Barbara G, De Giorgio R, Stanghellini V, et al. New pathophysiological mechanisms in irritable bowel syndrome. Aliment Pharmacol Ther 2004;20(Suppl 2): $1-9$.

14 Drossman DA, Camilleri M, Mayer EA, et al. AGA technical review on irritable bowel syndrome. Gastroenterology 2002;123:2108-2131.

15 McKenna BJ. Is it really colitis? Dealing with the nearly normal colonic biopsy and variations of microscopic colitis. Pathol Case Rev 2004;9:106-114.

16 Dunlop SP, Jenkins D, Spiller RC. Age-related decline in rectal mucosal lymphocytes and mast cells. Eur J Gastroenterol Hepatol 2004;16:1011-1015.

17 Levin DS, Haggitt RC. Colon. In: Sternberg SS (ed). Histology for Pathologists, 2nd edn. Lippincott-Raven: Philadelphia, PA, 1997, pp 519-537.

18 Bejarano PA, Aranda-Michel J, Fenoglio-Preiser C. Histochemical and immunohistochemical characterization of foamy histiocytes (muciphages and xanthelasma) of the rectum. Am J Surg Pathol 2000;24: 1009-1015.

19 Leriche M, Devroede G, Sanchez G, et al. Changes in the rectal mucosa induced by hypertonic enemas. Dis Colon Rectum 1978;21:227-236.

20 Zwas FR, Cirillo NW, el-Serag HB, et al. Colonic mucosal abnormalities associated with oral sodium phosphate solution. Gastrointest Endosc 1996;43: 463-466.

21 Hixson LJ. Colorectal ulcers associated with sodium phosphate catharsis. Gastrointest Endosc 1995;42: 101-102.

22 Driman DK, Preiksaitis HG. Colorectal inflammation and increased cell proliferation associated with oral sodium phosphate bowel preparation solution. Hum Pathol 1998;29:972-978.

23 Chadwick VS, Chen W, Shu D, et al. Activation of the mucosal immune system in irritable bowel syndrome. Gastroenterology 2002;122:1778-1783.

24 Dunlop SP, Jenkins D, Neal KR, et al. Relative importance of enterochromaffin cell hyperplasia, anxiety, and depression in postinfectious IBS. Gastroenterology 2003;125:1651-1659.

25 Dunlop SP, Jenkins D, Spiller RC. Distinctive clinical, psychological, and histological features of postinfective irritable bowel syndrome. Am J Gastroenterol 2003;98:1578-1583.

26 Salzmann JL, Peltier-Koch F, Bloch F, et al. Morphometric study of colonic biopsies: a new method of estimating inflammatory diseases. Lab Invest 1989;60: 847-851. 
27 Tornblom H, Lindberg G, Nyberg B, et al. Fullthickness biopsy of the jejunum reveals inflammation and enteric neuropathy in irritable bowel syndrome. Gastroenterology 2002;123:1972-1979.

28 Hiatt RB, Katz L. Mast cells in inflammatory conditions of the gastrointestinal tract. Am J Gastroenterol 1962;37:541-545.

29 Park CH, Joo YE, Choi SK, et al. Activated mast cells infiltrate in close proximity to enteric nerves in diarrhea-predominant irritable bowel syndrome. J Korean Med Sci 2003;18:204-210.

30 Barbara G, Stanghellini V, De Giorgio R, et al. Activated mast cells in proximity to colonic nerves correlate with abdominal pain in irritable bowel syndrome. Gastroenterology 2004;126:693-702.

31 O’Sullivan M, Clayton N, Breslin NP, et al. Increased mast cells in the irritable bowel syndrome. Neurogastroenterol Motil 2000;12:449-457.

32 Weston AP, Biddle WL, Bhatia PS, et al. Terminal ileal mucosal mast cells in irritable bowel syndrome. Dig Dis Sci 1993;38:1590-1595.

33 Talley NJ, Butterfield JH. Mast cell infiltration and degranulation in colonic mucosa in the irritable bowel syndrome. Am J Gastroenterol 1996;91:1675-1676.

34 Irvine EJ, Gaebel K, Driman D, et al. Mucosal mast cells (MMC) numbers are normal in biopsies from patients with Irritable Bowel Syndrome (IBS) [abstract]. Gastroenterology 1995;108:A860.

35 Pang X, Boucher W, Triadafilopoulos G, et al. Mast cell and substance P-positive nerve involvement in a patient with both irritable bowel syndrome and interstitial cystitis. Urology 1996;47:436-438.

36 Barbara G, Stanghellini V, De Giorgio R, et al. Neuroimmune relationships in the colonic mucosa of irritable bowel syndrome patients [abstract]. Neurogastroenterol Motil 2000;12:A272.

37 Barbara GC, De Giorgio R, Cogliandro L, et al. Intestinal neuro-immune interactions in irritable bowel syndrome are gender dependent [abstract]. Digestive Liver Diseases 2001;33:A31.

38 Spiller RC. Neuropathology of IBS? Gastroenterology 2002;123:2144-2147.

39 Collins SM, Barbara G. East meets West: infection, nerves, and mast cells in the irritable bowel syndrome. Gut 2004;53:1068-1069.

40 Gwee KA, Collins SM, Read NW, et al. Increased rectal mucosal expression of interleukin 1 beta in recently acquired post-infectious irritable bowel syndrome. Gut 2003;52:523-526.

41 Collins SM. A case for an immunological basis for irritable bowel syndrome. Gastroenterology 2002;122: 2078-2080.

42 Collins SM, Barbara G, Vallance B. Stress, inflammation and the irritable bowel syndrome. Can J Gastroenterol 1999;13(Suppl A):47A-49A.

43 Collins SM, McHugh K, Jacobson K, et al. Previous inflammation alters the response of the rat colon to stress. Gastroenterology 1996;111:1509-1515.

44 Collins SM, Vallance B, Barbara G, et al. Putative inflammatory and immunological mechanisms in functional bowel disorders. Baillieres Best Pract Res Clin Gastroenterol 1999;13:429-436.

45 Barbara G, De Giorgio R, Stanghellini V, et al. A role for inflammation in irritable bowel syndrome? Gut 2002;51(Suppl 1):i41-i44.

46 Bueno L, Fioramonti J, Delvaux M, et al. Mediators and pharmacology of visceral sensitivity: from basic to clinical investigations. Gastroenterology 1997;112: 1714-1743.

47 Vergnolle N, Bunnett NW, Sharkey KA, et al. Proteinase-activated receptor-2 and hyperalgesia: a novel pain pathway. Nat Med 2001;7:821-826.

48 Suzuki R, Furuno T, McKay DM, et al. Direct neuritemast cell communication in vitro occurs via the neuropeptide substance P. J Immunol 1999;163: 2410-2415.

49 Talley NJ, Zinsmeister AR, Melton 3rd LJ. Irritable bowel syndrome in a community: symptom subgroups, risk factors, and health care utilization. Am J Epidemiol 1995;142:76-83.

50 Gue M, Del Rio-Lacheze C, Eutamene H, et al. Stressinduced visceral hypersensitivity to rectal distension in rats: role of CRF and mast cells. Neurogastroenterol Motil 1997;9:271-279.

51 Bearcroft CP, Perrett D, Farthing MJ. Postprandial plasma 5-hydroxytryptamine in diarrhoea predominant irritable bowel syndrome: a pilot study. Gut 1998; 42:42-46.

52 Houghton LA, Atkinson W, Whitaker RP, et al. Increased platelet depleted plasma 5-hydroxytryptamine concentration following meal ingestion in symptomatic female subjects with diarrhoea predominant irritable bowel syndrome. Gut 2003;52: 663-670.

53 De Ponti F, Tonini M. Irritable bowel syndrome: new agents targeting serotonin receptor subtypes. Drugs 2001;61:317-332.

54 Kapraali M, Johansson O, Uribe A. Endogenous prostaglandins are physiological regulators of endocrine cells in the gastroduodenal mucosa of the rat. Regul Pept 1999;83:105-116.

55 Wheatcroft J, Wakelin D, Smith A, et al. Enterochromaffin cell hyperplasia and decreased serotonin transporter in a mouse model of postinfectious bowel dysfunction. Neurogastroenterol Motil 2005;17: 863-870.

56 Gershon MD. Review article: serotonin receptors and transporters-roles in normal and abnormal gastrointestinal motility. Aliment Pharmacol Ther 2004; 20(Suppl 7):3-14

57 Baker DE. Rationale for using serotonergic agents to treat irritable bowel syndrome. Am J Health Syst Pharm 2005;62:700-711.

58 Friedel D, Thomas R, Fisher RS. Ischemic colitis during treatment with alosetron. Gastroenterology 2001;120:557-560.

59 Cole JA, Cook SF, Sands BE, et al. Occurrence of colon ischemia in relation to irritable bowel syndrome. Am J Gastroenterol 2004;99:486-491.

60 Stead RH, Kosecka-Janiszewska U, Oestreicher AB, et al. Remodeling of B-50 (GAP-43)- and NSE-immunoreactive mucosal nerves in the intestines of rats infected with Nippostrongylus brasiliensis. J Neurosci 1991;11:3809-3821.

61 Peters EM, Kuhlmei A, Tobin DJ, et al. Stress exposure modulates peptidergic innervation and degranulates mast cells in murine skin. Brain Behav Immun 2005;19:252-262.

62 Collins SM. The immunomodulation of enteric neuromuscular function: implications for motility and inflammatory disorders. Gastroenterology 1996;111: 1683-1699.

63 Latcham F, Merino F, Lang A, et al. A consistent pattern of minor immunodeficiency and subtle entero- 
pathy in children with multiple food allergy. J Pediatr 2003;143:39-47.

64 Wakefield AJ, Murch SH, Anthony A, et al. Ileallymphoid-nodular hyperplasia, non-specific colitis, and pervasive developmental disorder in children. Lancet 1998;351:637-641.

65 Furlano RI, Anthony A, Day R, et al. Colonic CD8 and gamma delta T-cell infiltration with epithelial damage in children with autism. J Pediatr 2001;138:366-372.

66 Ashwood P, Anthony A, Pellicer AA, et al. Intestinal lymphocyte populations in children with regressive autism: evidence for extensive mucosal immunopathology. J Clin Immunol 2003;23:504-517.

67 Ashwood P, Anthony A, Torrente F, et al. Spontaneous mucosal lymphocyte cytokine profiles in children with autism and gastrointestinal symptoms: mucosal immune activation and reduced counter regulatory interleukin-10. J Clin Immunol 2004;24:664-673.

68 Bryant DA, Mintz ED, Puhr ND, et al. Colonic epithelial lymphocytosis associated with an epidemic of chronic diarrhea. Am J Surg Pathol 1996;20: 1102-1109. 